\title{
What lessons can be learned from the perspective of the different actors in DM, especially from companies and social security organisations?
}

Thomas Geisen

North Western Switzerland University of Applied Sciences and Arts, Switzerland. thomas.geisen@fhnw.ch

Background: In the context of demographic change and lack of qualified workers the preservation of the internal labour force gains importance for the companies. At the same time private and public income security organisations are increasingly interested in achieving better return to work out-comes in order to reduce their costs. Research shows that a functioning DM leads to more effective return to work in companies.

Objectives: The interaction between the welfare state, the companies and private insurers is quite different in each county and highly depending and formed by the established welfare state scheme. In the presentation we will describe the interplay between the different stakeholders in DM for the situation in Switzerland and derive specific challenges and opportunities that arise from this set-ting. Methods: The presentation is based on findings from two studies (2008 and 20013) on disability management in private companies in Switzerland relying on qualitative and quantitative data. The results of these studies will be enriched with first findings from an ongoing international study that compares DM programmes in Switzerland, Australia, Canada and China (2013-2015).

Findings: The cooperation between social insurances and the companies could be improved in various ways. Most important is the improvement of informal and institutional communication between the company and the social insurances representatives. But there is also a need to more effective and efficient cooperation in improving rehabilitation processes and the processes of work-place integration. In general the studies show not only that organisational development is needed but also the qualification of DMP's, as key factors in the processes, need to be improved.

Discussion: We will present findings from a study on DM programmes in private companies from 2008, from a study on DM activities in small and medium sized companies 2013 and first results from an on-going international comparative research project mentioned above. What lessons can be learned from the perspective of the different actors in DM, especially from companies and social security organisations?

Conclusion: Companies, private insurers and social security organizations will gain new insights into challenges and opportunities of DM. The findings will help to enhance collaboration between these different stakeholders and might provide important insight to improve and enhance existing DM programmes. 